\title{
The functional role of the CARM1-SNF5 complex and its associated HMT activity in transcriptional activation by thyroid hormone receptor
}

\author{
Hyo-Kyoung Choi ${ }^{1,2}$, Kyung-Chul Choi ${ }^{1,2}$, \\ So-Young $\mathrm{Oh}^{3}$, Hee-Bum Kang ${ }^{1,2}$, Yoo-Hyun Lee, \\ Seungjoo Haam ${ }^{4}$, Yong-Ho Ahn ${ }^{1}$, Kyung-Sup Kim ${ }^{1,2}$, \\ Kunhong $\mathrm{Kim}^{1,2,5}$ and Ho-Geun Yoon ${ }^{1,2,5}$ \\ ${ }^{1}$ Department of Biochemistry and Molecular Biology \\ Center for Chronic Metabolic Disease Research \\ ${ }^{2}$ Brain Korea 21 Project for Medical Sciences \\ Yonsei University College of Medicine \\ Seoul 120-752, Korea \\ ${ }^{3}$ Howard Hughes Medical Institute \\ Program in Gene Function and Expression \\ University of Massachusetts Medical School \\ Worcester, MA 01605, USA \\ ${ }^{4}$ Department of Chemical Engineering \\ College of Engineering \\ Yonsei University \\ Seoul 120-749, Korea \\ ${ }^{5}$ Corresponding authors: Tel, 82-2-2228-1683; \\ Fax, 82-2-312-5041; E-mail, yhgeun@yumc.yonsei.ac.kr (for H.G.Y.) \\ Tel, 82-2-2228-1680; Fax, 82-2-312-5041; E-mail: kimkh34@yumc. \\ yonsei.ac.kr (for K.H.K.)
}

Accepted 27 June 2007

Abbreviations: CARM1, coactivator associated arginine methyltransferase 1; HMT, histone methyltransferase; siRNA, small interfering RNA; SNF5, SWI/SNF complex component 5; TR, thyroid hormone receptor; $\mathrm{RXR}$, retinoid $\mathrm{X}$ receptor

\begin{abstract}
We have investigated the function and mechanisms of the CARM1-SNF5 complex in T3-dependent transcriptional activation. Using specific small interfering RNAs (siRNA) to knock down coactivators in HeLa $\alpha 2$ cells, we found that coactivator associated arginine methyltransferase 1 (CARM1) and SWI/SNF complex component 5 (SNF5) are important for T3-dependent transcriptional activation. The CARM1- SWI/SNF chromatin remodeling complex serves as a mechanism for the rapid reversal of $\mathrm{H} 3-\mathrm{K} 9$ methylation. Importantly, siRNA treatment against CARM1 and/or SNF5 increased the recruitment of HMTase G9a to the type 1 deiodinase (D1) promoter even with T3. Knocking-
\end{abstract}

down either CARM1 or SNF5 also inhibited the downregulation of histone macroH2A, which is correlated with transcriptional activation. Finally, knocking down CARM1 and SNF5 by siRNA impaired the association of these coactivators to the D1 promoter, suggesting functional importance of CARM1- SNF5 complex in T3-dependent transcriptional activation.

Keywords: coactivator-associated arginine methyltransferase 1; RNA, small interfering; SMARCB1 protein, human; transcription factors

\section{Introduction}

Thyroid hormone receptors (TRs) can bind to target promoter as monomers, homodimers, or heterodimers with retinoid $X$ receptor (RXR) (An et al., 1997). TRs can positively or negatively regulate transcription dependent on the presence or absence of hormones and the presence of a positive or negative thyroid hormone response element (TRE vs nTRE) in its target genes (O'Shea and Williams, 2002). In the absence of ligand, TR/RXR heterodimers bind to TREs and actively repress transcription. The repression by unliganded $T R / R X R$ is largely mediated through the recruitment of corepressor proteins such as silencing mediator for retinoic acid receptor and thyroidhormone receptor (SMRT) and nuclear hormone receptor co-repressor (N-CoR) (Guenther et al., 2000; Yoon et al., 2003a). Binding of T3 to TR is believed to induce conformational changes of the receptor, which in turn lead to the release of corepressors and the recruitment of coactivators. Compared to repression by unliganded TR, transcriptional activation by liganded TR is much more complex. First, hormone-dependent activation appears to involve a significantly larger number of cofactors. The current list of nuclear hormone receptor (NR) coactivators contains more than 200 proteins (Narr et al., 2001, McKenna and O'Malley, 2002). Although it is extremely unlikely that all these putative coactivators are required for activation by nuclear receptors, studies from many laboratories provide evidence that many coactivators are required and act in concert to mediate transcriptional activation by nuclear receptors 
(Rosenfield and Glass, 2001; Lee et al., 2002). A number of coactivators contain the signature motif LXXLL sequences ( $X$, any amino acid) that interact with the ligand-binding domain of NRs in a liganddependent manner (Heery et al., 1997; Mclnerney et al., 1998).

Based on the studies of CREB-binding protein (CBP) and E1A-binding protein, 300-kDa (p300) knockout mice (Kawasaki et al., 1998; Yao et al., 1998), we know that CBP and p300 are critically important for activation by RAR. Their intrinsic histone acetyltrasferase (HAT) activity is likely critical for their ability to enhance activation by many NRs (Kraus et al., 1999; Yi et al., 2007), although in some cases the HAT activity of p300/CBP-associated factor (PCAF) may be preferentially used (Korzus et al., 1998). The general requirement for $\mathrm{CBP} / \mathrm{p} 300$ is consistent with the notion that histone acetylation is critically important for transcriptional activation from repressive chromatin. In this regard, the major function of the steroid hormone receptor coactivator (SRC) family, which interacts directly with liganded receptors, is believed to serve as bridging molecules to recruit CBP/p300 and other coactivators such as CARM1 (Xu and Li, 2003)

In addition to histone acetylation, histone methylation by CARM 1 has been shown to facilitate hormone-dependent activation by NRs (Chen et al., 1999; Wang et al., 2001). CARM1 methylates histone $\mathrm{H} 3$ at Arg 2, 17 and 26 (Strahl et al., 2001). CARM1 cooperates synergistically with the SRC family of coactivators and CBP/p300 to enhance transcriptional activation by NRs in transient transfection assays (Chen et al., 2000). In addition, CARM1 associates with the SWI/SNF complex and this association seems to enhance the HMT activity of CARM1 (Xu et al., 2004). Finally, CARM1 null mice die perinatally and estrogen-responsive gene expression is aberrant in Carm1-/fibroblasts and embryos, thus indicating a critical role for CARM1 in mediating transcriptional regulation by estrogen receptors (Yadav et al., 2003). However, given that CARM1 can methylate not only histones but also other proteins, it is not clear whether histone methylation by CARM1 is critically important for transcriptional activation by NRs. In addition to the enzymes that covalently modify histones, the ATP-dependent chromatin remodeling factor, SWI/SNF, also has a critical role in transcriptional activation by NRs (Chiba et al., 1994; Wong et al., 1995). Human SWI/SNF is heterogeneous and known to contain either Brm or its related protein Brg1, the ATPase subunits of the complexes (Wang et al., 1996). In transient transfection and in vitro transcription assays, both $\mathrm{hBrm}$ and $\mathrm{hBrg} 1$ can facilitate transcriptional activation by glucocorticoid receptor (GR), estrogen receptor (ER), and retinoic acid receptor (RAR) (Dilworth et al., 2000; DiRenzo et al., 2000). Accumulated evidence indicates that the coactivators CARM1 and ATP- dependent chromatin remodeling factor SWI/ SNF have critical functions in transcriptional activation by nuclear receptors. However, the underlying mechanism for requirement of various chromatin remodeling factors remains obscure.

In this study, we have investigated the function and mechanisms of the CARM1-SNF5 complex in T3-dependent transcriptional activation. We found that CARM1 and SNF5 to a large extent are important for T3-dependent transcriptional activation. The CARM1-SWI/SNF chromatin remodeling complex serves as a mechanism for the rapid reversal of H3-K9 methylation. Finally, knocking down CARM1 and SNF5 by siRNA impaired the association of coactivator P300 to the D1 promoter, suggesting functional importance of CARM1-SNF5 complex in T3-dependent activation.

\section{Materials and Methods}

\section{Cell culture and siRnA}

Cell culture and siRNA treatment were carried out essentially as previously described (Yoon et al., 2003b, 2005). For transfection of siRNAs, HeLa $\alpha 2$ cells were first cultured in DMEM supplemented with $10 \%$ charcoal-stripped serum for three days and then transfected at a cell confluency of $\sim 40-50 \%$ using Lipofectamine 2000 with amounts of siRNA as indicated by the manufacturer's instructions. Two days after transfection, cells were collected and processed for western analysis, RT-PCR, or chromatin immunoprecipitation (ChIP) as indicated. For experiments with T3, $\alpha 2$ cells were initially seeded at a density of $4 \times 10^{5}$ cells/100 mm tissue culture dish. After a 24-h incubation, the culture media was replaced with DMEM with $10 \%$ charcoal-stripped FCS (CS-FCS) (Gemini Bio-Products) for three days followed by replacement with fresh CS-FCS supplemented with $10 \mathrm{nM} \mathrm{T3}$ for up to six $\mathrm{h}$. For the experiments involving both siRNAs and T3, T3 was added two days after siRNA transfection and incubated for one $\mathrm{h}$ or as indicated in the ChIP assays and six $\mathrm{h}$ for RT-PCR analysis. The siRNAs for SNF5, CBP, PCAF, PRMT5, BRG1, ASC2 and Brm were chemically synthesized (Dharmacon Research, Lafayette, Co), de-protected and annealed according to the manufacturer's instruction. The siRNA sequences used are as follows: SNF5-1, AAUGUUCCGAGGUUCUCUGUA; SNF 5-2, AAUGGCAAC- 
GAUGAGAAGUAC; CBP-1, AAACTCGTCCAAGCCATCTTCA; CBP-2, AAACGGAGGTCGCGTTTACAT; CBP-3, AACGGAGGTCGCGTTTACATA; PCAF-1, AACCTGTGGTTGAAGGCTCTT; PCAF2, AATCGCCGTGAAGAAAGCGCA; PCAF-3, AAACGAACTCTAATCCTCACT; PRMT5-1, AACGTGTATGGCTTCGACATG; PRMT 5-2, AAAGGAGGTGGACATCTATAC; BRG1-1, AATGCCAAGCAAGATGTCGAT; BRG1-2, AAATACCTCAGGCTTGATGGA; BRG1-3, AAATCGAGAAGGAGGATGACA; ASC2-1, AAGTGGTCCAGGAATAATAAG; ASC22, AATAAGGATGTCACGCTAACG; ASC2-3, AAAGTTACAGGCTCTCTTGAG; Brm- 1, AACGGAATCTTAGCCGATGAA; Brm-2, AAAATTGAGAGCGACTAATCA; Brm-3, AAGAATTACCAGAATACTATG. The pre-designed siRNAs for p300, CARM1, p68, SRC1, SRC2 and SRC3 were obtained from Dharmacon. The efficiency of siRNA knockdown was determined by western analysis using the corresponding specific antibodies.

\section{ChIP assays}

For ChIP assays, we first isolated chromatin as previously described (Yoon et al., 2005). In brief, approximately $2 \times 10^{9} \alpha 2$ cells in $150-\mathrm{mm}$ dishes were first treated with PBS containing 1\% formaldehyde for $10 \mathrm{~min}$, washed twice with PBS, and then incubated with $100 \mathrm{mM}$ Tris $(\mathrm{pH} \mathrm{9.4)}$ and $10 \mathrm{mM}$ DTT at $30^{\circ} \mathrm{C}$ for $15 \mathrm{~min}$. The cells were then rinsed twice with PBS and resuspended in $600 \mu \mathrm{l}$ of Sol A buffer [10 mM Hepes ( $\mathrm{pH} 7.9$ ), $0.5 \% \mathrm{NP}-40,1.5 \mathrm{mM} \mathrm{MgCl}, 10 \mathrm{mM} \mathrm{KCl}, 0.5 \mathrm{mM}$ DTT] by pipetting. After a short spin (5 min at 3,000 $\mathrm{rpm})$, the pellets were resuspended in Sol B [20 $\mathrm{mM}$ Hepes ( $\mathrm{pH} 7.9$ ), 25\% glycerol, 0.5\% NP-40, $0.42 \mathrm{M} \mathrm{NaCl}, 1.5 \mathrm{mM} \mathrm{MgCl}$, $0.2 \mathrm{mM}$ EDTA] containing protease inhibitors followed by vigorous pipetting in order to extract the nuclear proteins. After centrifugation at $13,000 \mathrm{rpm}$ for $30 \mathrm{~min}$, the nuclear pellets were resuspended in IP buffer $(1 \%$ Triton X-100, 2 mM EDTA, 20 mM Tris- $\mathrm{HCl}$ pH 8.0, $150 \mathrm{mM} \mathrm{NaCl}$ and protease inhibitors) and sonicated to break the chromatin into fragments with an average length of $0.5-1 \mathrm{~kb}$. The ChIP assays were then performed with the indicated antibodies essentially as previously described, though, SDS was omitted in all buffers. The antibodies against acetylated p300, G9a, Suv39H1, TR, Dimethyl R17-H3, AcH3, and TrimethylK9-H3 were purchased from Upstate Biotechnology. The antibody against LSD1 and Brm1 were obtained from Santa Cruz Biotechnology. The antibodies against JHDM2A, SNF5 and macroH2A were kindly provided by Dr. Wong J (East China Univesity). The antibodies against SRC1, SRC2,
SRC3, p300 and SNF5 were kindly provided by Dr. Qin J (Baylor College of Medicine). The primers used for ChIP analysis were previously described (Yoon et al., 2005).

\section{RNA extraction and RT-PCR}

Total RNA was isolated from $\alpha 2$ cells using an RNeasy Mini kit (Qiagen, Tokyo, Japan), according to the manufacturer's specifications. Total RNA from each sample was reverse-transcribed with random primers using a StrataScript ${ }^{\mathrm{TM}}$ reverse transcriptase kit (Stratagene, La Jolla, CA) followed by quantitative PCR analysis. Primers for ADRB2 amplification were 5'-GGATCGCTACTTTGCCATTACTTCACCTTTCA-3' and 5'-CCCCGTCCGCCCATCCTGCTCCAC-3'. Primers for BCL3 amplification were 5'-ACGCCGTGGAAAACAACAGCCTTAGCAT-3' and 5'-GAGCGGCGTGTCGTTGTGGCAGTTCTTGAG-3'. Primers for FAS amplification were 5'-CATCGGGCACGTGGGCATTTTG-3' and 5'-GGTCCCGCTGGCTGTCCCTGTCC3'. Primers for D1 amplification were 5'-AACCCCCATTTCAGCCACGACAAC-3' and 5'-ATTCAGCACCAGTGGCCTATTACCTT-3'. Primers for GAPDH amplification were 5'-CGCGGGGCTCTCCAGAACATCATCC-3' and 5'-CTCCGACGCCTGCTTCACCACCTTCTT-3'. PCR products were separated by agarose gel electrophoresis and visualized by ethidium bromide staining.

\section{Real-time PCR analysis}

The RT-PCR analysis and quantification were performed with Taqman One-Step RT-PCR Master Mix Reagents or SYBR Green PCR Master mix Reagents [depending on the target RNA (see below)] on an ABI Prism 7700 Sequence Detection System (Applied biosystem, Foster city, CA). The singularity and specificity of amplifications were checked by Dissociation Analysis Software. All samples were normalized to 18S rRNA (Applied biosystem). Primer sequences for amplification of the D1 RNA used in qRT-PCR were ( $F$ 5'-AGCCCATCTACTGCTGGCC-3', R 5'-TGTAGTTCCAAGGGCCAGATTT-3', and probe 5'-FAM-AGGCTCTACATAATCCAGGAGGGCAGGA-TAMRA-3').

All reactions were performed in triplicate. Relative expression levels and SDs were calculated using the comparative method. 


\section{Results}

\section{Both CARM1 and SNF5 are important for T3-dependent activation of the D1 gene}

Transcription is a complex, multi-step process involving a large number of cofactors that affect chromatin remodeling, formation of RNA polymerase II preinitiation complex (PIC), transcription elongation and the RNA processing (Levine and Tjian, 2003). To successfully transcribe a gene, each essential cofactor must be recruited to the promoter in a dynamic fashion to fulfill its specific function and allow the next step to proceed.

Using Xenopus laevis oocytes, we previously showed that transcriptional activation by liganded TR is coupled with not only increased acetylation on $\mathrm{H} 3$ and $\mathrm{H} 4$ but also a robust decrease in $\mathrm{H} 3-\mathrm{K} 9$ methylation. Furthermore, the pronounced effect on histone acetylation was repeatedly observed upon T3 treatment, presumably as a combined result of corepressor complex dissociation and

A
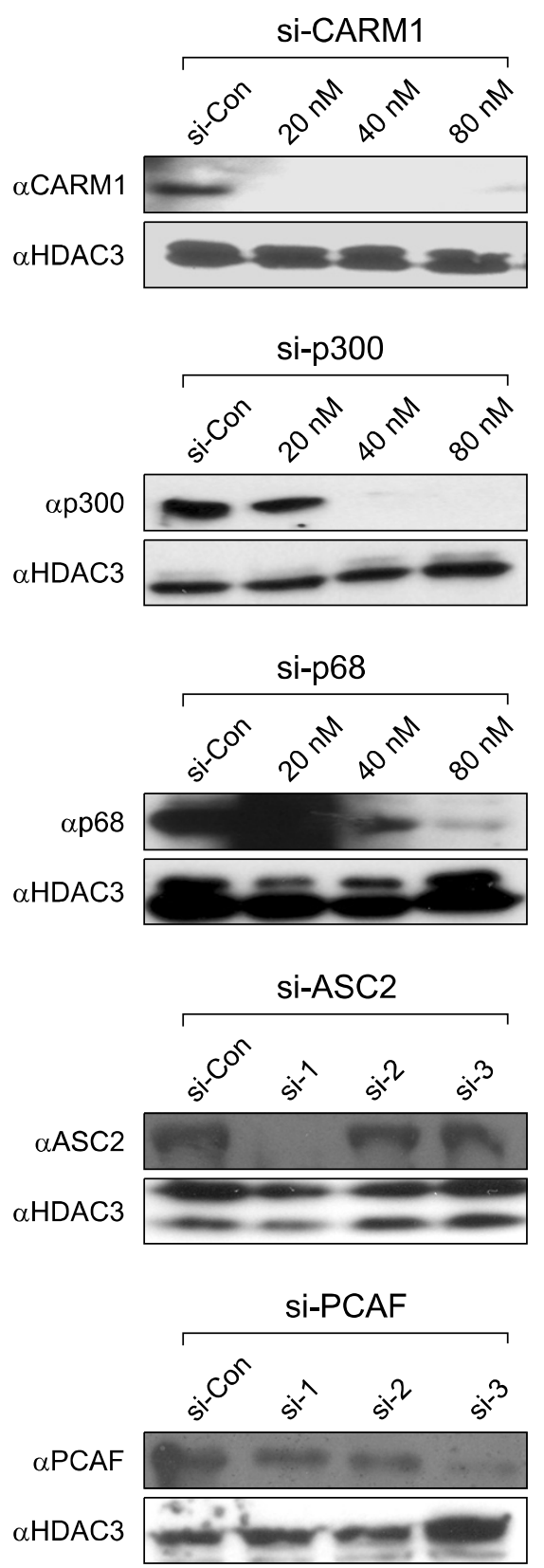
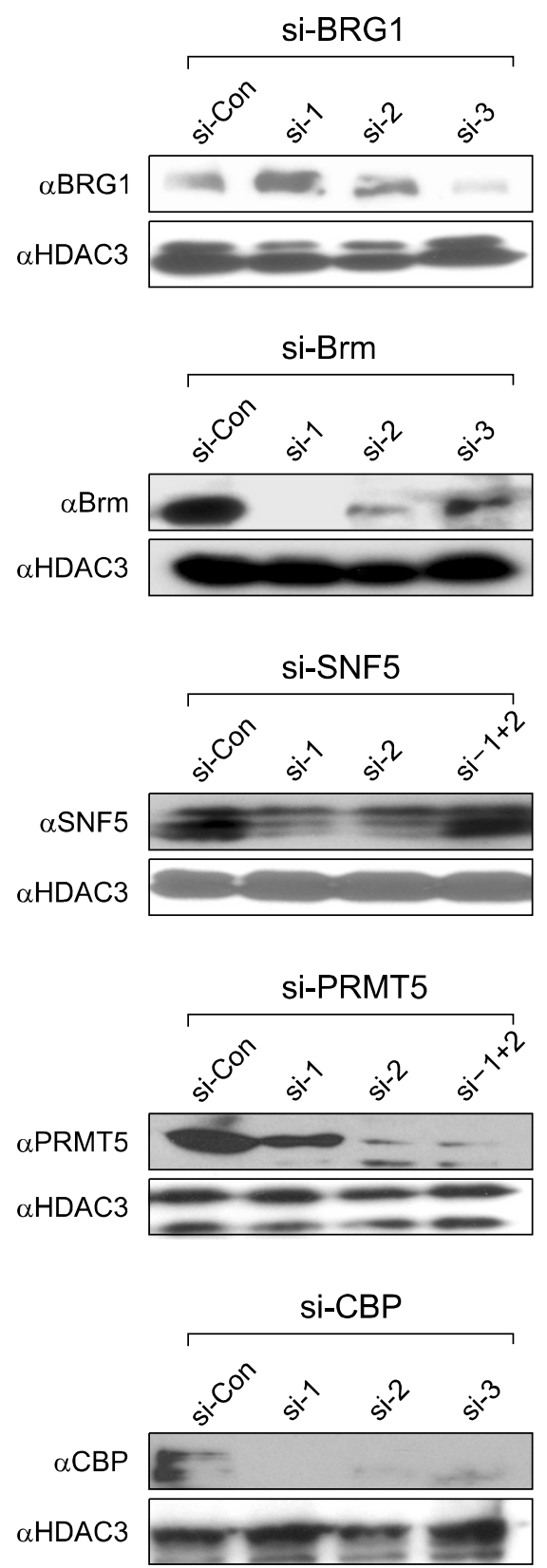

Figure 1. Effect of siRNAs against coactivators on T3-dependent transcription. (A) HeLa $\alpha 2$ cells were transfected with control (scrambled) siRNA or siRNA against coactivators at the concentration indicated. Three days after treatment, whole cell extracts were prepared and the levels of each protein were determined by western blot. Western blot results for HDAC3 served as specificity controls. 
B

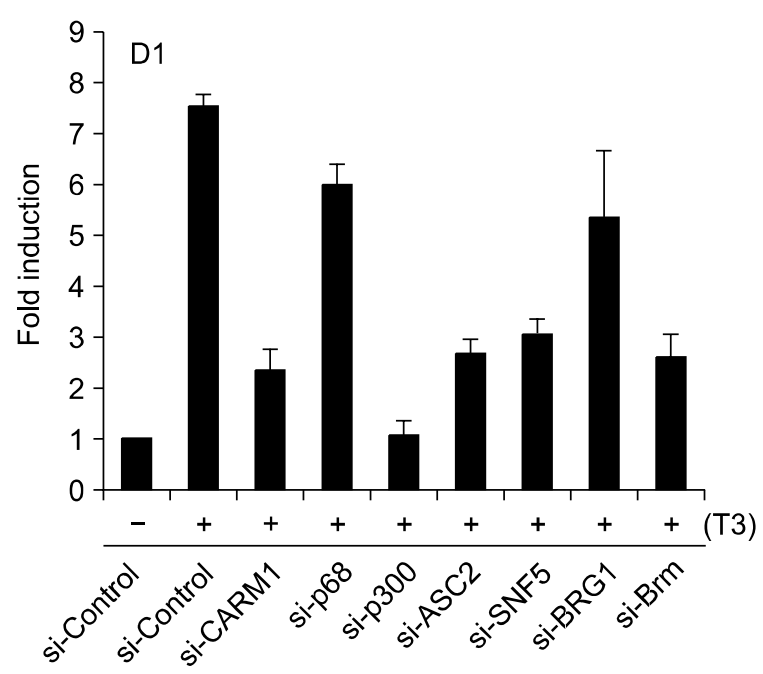

C
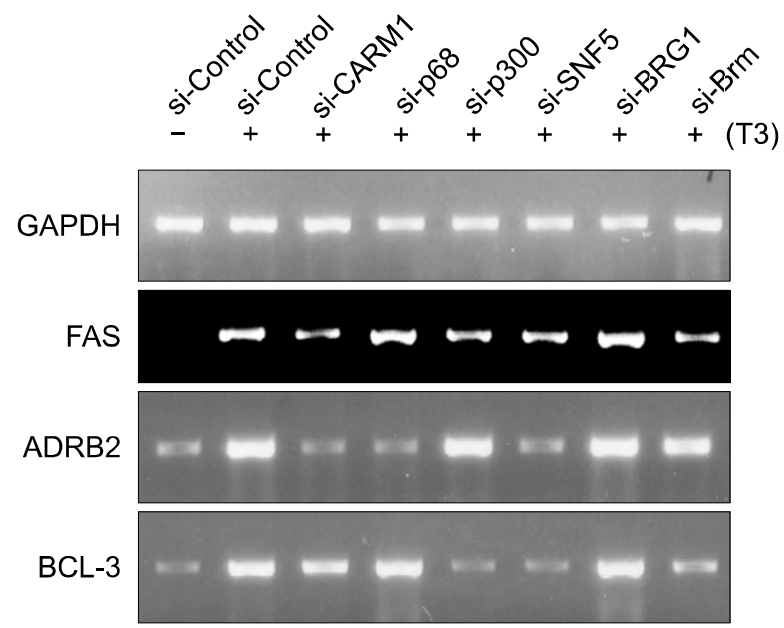

Figure 1. Continued. (B) The effect on D1 transcriptional activation was analyzed by quantitative real-time PCR analyses. After treatment with siRNAs as indicated, the cells were treated with T3 for six $h$. (C) Total RNA was prepared from each sample and the levels of FAS, ADRB2, and BCL3 were analyzed by semi-quantitative PCR. The real time data in (B) was normalized against $18 \mathrm{~S}$ rRNA. The level of actin mRNA served as a control in (C). The results are shown as means \pm SD calculated from three independent experiments.

subsequent active recruitment of coactivators by liganded TR (Li et al., 2002).

To address which coactivators are required for dynamic regulation between acetylation and K9-methylation of $\mathrm{H} 3$, we first developed specific siRNAs against various coactivators. To test the effect and specificity of siRNA constructs, HeLa $\alpha 2$ cells were transfected with three different siRNAs per target coactivator. Two days after transfection, HeLa $\alpha 2$ cells were collected, and the effect of siRNA treatment was analyzed by western blotting. Each siRNA treatment clearly silenced the expression of their corresponding target proteins. The siRNAs against CARM1, p300 and Brm worked more efficiently than the siRNAs against p68, BRG1 and SNF5 (Figure 1A). As a control, we measured the levels of HDAC3, a corepressor for nuclear receptors, which showed no significant change after any siRNA treatment. In fact, we tested and found none of the siRNAs used had any significant cross effect. For instance, siRNA against BRG1 selectively reduced the level of BRG1 protein but had no effect on SNF5, and vice versae (data not shown).

Having established the specific 'knock-down' effects caused by each siRNA, we next tested the effect of these specific siRNAs on TR activation. To assess which coactivators are required for T3dependent activation, we examined the effect of knocking down each of them on D1 expression, the TR target gene in HeLa $\alpha 2$ cells. For this purpose, HeLa $\alpha 2$ cells were treated with siRNA specifically against coactivators and a control siRNA as shown in Figure 1B. Three days after siRNA treatment, T3 $(50 \mathrm{nM})$ was added to the tissue cultures and incubated for an additional six h. Cells were then harvested and the level of D1 gene expression was determined by quantitative real time PCR. In each experiment, the effectiveness of the siRNA knock-down was confirmed by western analysis. Importantly, knock-down of CARM1 and SNF5 significantly crippled T3-dependent activation of the D1 gene, indicating that CARM1 and SNF5 are critically important for transcriptional activation by TR (Figure 1B). To assess whether CARM1 and SNF5 are generally required for T3-dependent activation, we tested the effect of knocking down CARM1 and SNF5 on the expression of FAS, ADRB2, and BCL3, another TR target genes in HeLa $\alpha 2$ cells. Three days after siRNA treatment, the cells were treated with $10 \mathrm{nM}$ T3 for six $h$, and the levels of FAS, ADRB2 and BCL3 gene expression were determined by RT-PCR. While treatments with siRNAs against coactivators did not alter the transcription level of the GAPDH gene, a significant effect was observed for most of the TR target genes (Figure $1 \mathrm{C})$. In this case, the knockdown of CARM1 and SNF5 in general led to a repression of liganded TR-mediated transcription from all three TR target genes, although the extent of decreased expression was gene-dependent. Together, we conclude, at least for the genes we have tested, that CARM1 and SNF5 are generally required for 

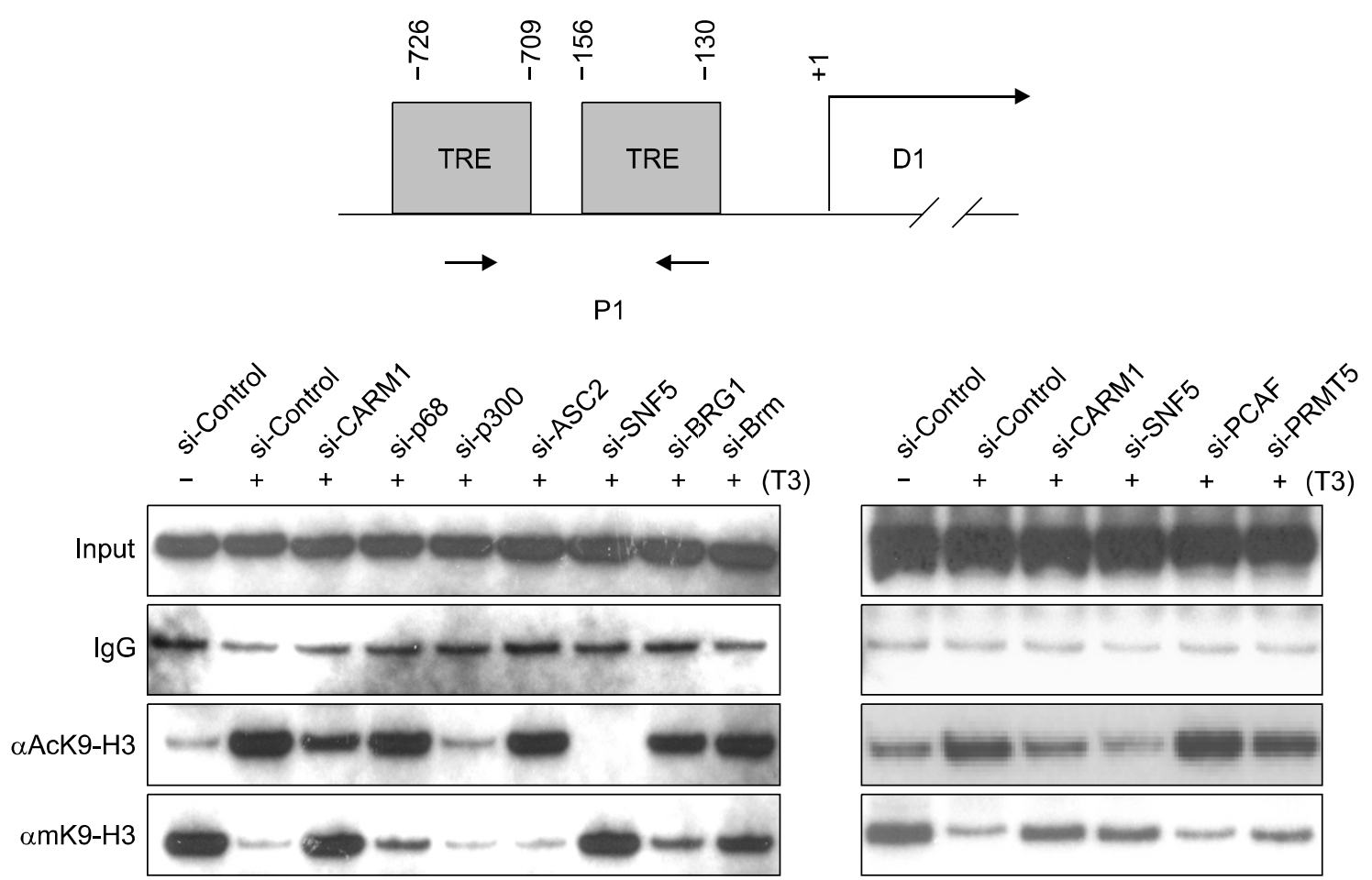

Figure 2. Both CARM1 and SNF5 are required for the rapid reversal of H3-K9 methylation. HeLa $\alpha 2$ cells were transfected with control (scrambled) siRNA or siRNA against coactivators at the concentration indicated. The cells were then treated with T3 for $6 \mathrm{~h}$, after which the levels of acetylated and methylated lysine 9 of H3 were determined by ChIP assays using the antibodies indicated.

T3-dependent transcriptional activation in mammalian cells.

\section{Chromatin remodeling by CARM1-SWI/SNF is essential for reversal of $\mathrm{H} 3-\mathrm{K} 9$ methylation}

The presence of $\mathrm{K} 9$ methylated $\mathrm{H} 3(\mathrm{mH} 3-\mathrm{K} 9)$ in genes repressed by unliganded TR raises question as to how the liganded receptor overcomes this repressive activity during T3-dependent activation. As shown in Figure 2, we found by ChIP assay that the level of $\mathrm{mH} 3-\mathrm{K} 9$ in the $\mathrm{D} 1$ promoter was rapidly decreased upon T3 treatment. As knock-down of CARM1 and SNF5 significantly crippled T3-dependent activation of the D1 gene, we next determined whether chromatin remodeling by CARM1-SWI/ SNF is essential for reversal of $\mathrm{H} 3-\mathrm{K} 9$ methylation. To do this, HeLa $\alpha 2$ cells were treated with siRNA specifically against coactivators and a control siRNA as shown in Figure $1 \mathrm{~A}$. Three days after siRNA treatment, T3 $(10 \mathrm{nM})$ was added to the tissue cultures and incubated for an additional six $\mathrm{h}$. Cells were then harvested and histone modification was determined by ChIP assays. ChIP results showed that treatment of cells with $\mathrm{T} 3$ led to a significant increase of acetylated $\mathrm{H} 3$ levels. $\mathrm{T} 3$ treatment also resulted in a reduction of $\mathrm{mH} 3-\mathrm{K} 9$ in the D1 target. Interestingly, knocking down either CARM1 or SNF5 led to a decrease in histone acetylation even of T3 (Figure 2). Knocking-down P300 induced a robust decrease of acetylation, as expected. Taken together, these results support the idea that chromatin remodeling by CARM1SWI/SNF serves as a mechanism for the rapid reversal of $\mathrm{H} 3-\mathrm{K} 9$ methylation.

We next examined the relationship between knocking-down CARM1 or SNF5 and increase of methyl $\mathrm{K} 9-\mathrm{H} 3$ in the presence of T3. Chip patterns obtained on chromatin prepared from $\alpha 2$ cells, treated six $h$ with T3 after 3 days of hormonedeprivation, demonstrate that CARM1, SRC, p300 and SNF5 coexist with liganded-TR in the D1 promoter (Figure 3A). Furthermore, histone methyltransferase, G9a, but not Suv39H1, was recruited to $\mathrm{D} 1$ promoter in the absence of $\mathrm{T} 3$. In contrast to HMTase, the histone demethylase LSD1 and JHDM2A were not found on the D1 promoter regardless of $T 3$, implying the presence of other demethylases. Importantly, siRNA treatment against CARM1 and/or SNF5 increased the recruitment of HMTase, G9a compared with control siRNAs. This data suggested that the increased recruitment of G9a by knocking-down of CARM1SNF5 complex reversed severely T3-induced 
A

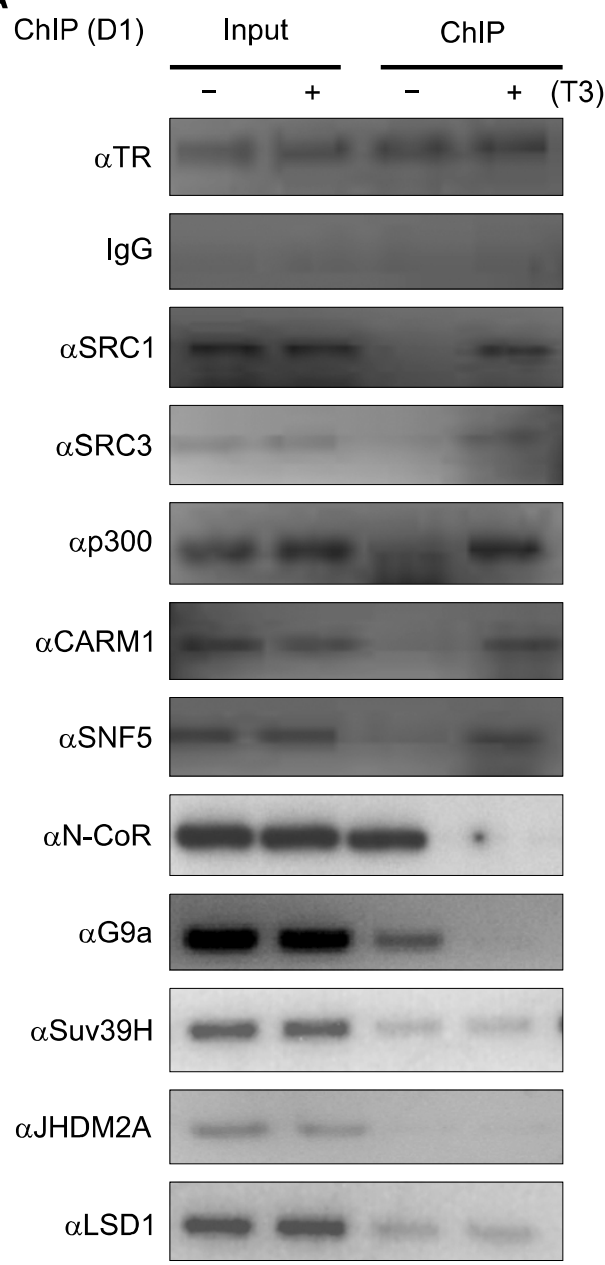

B

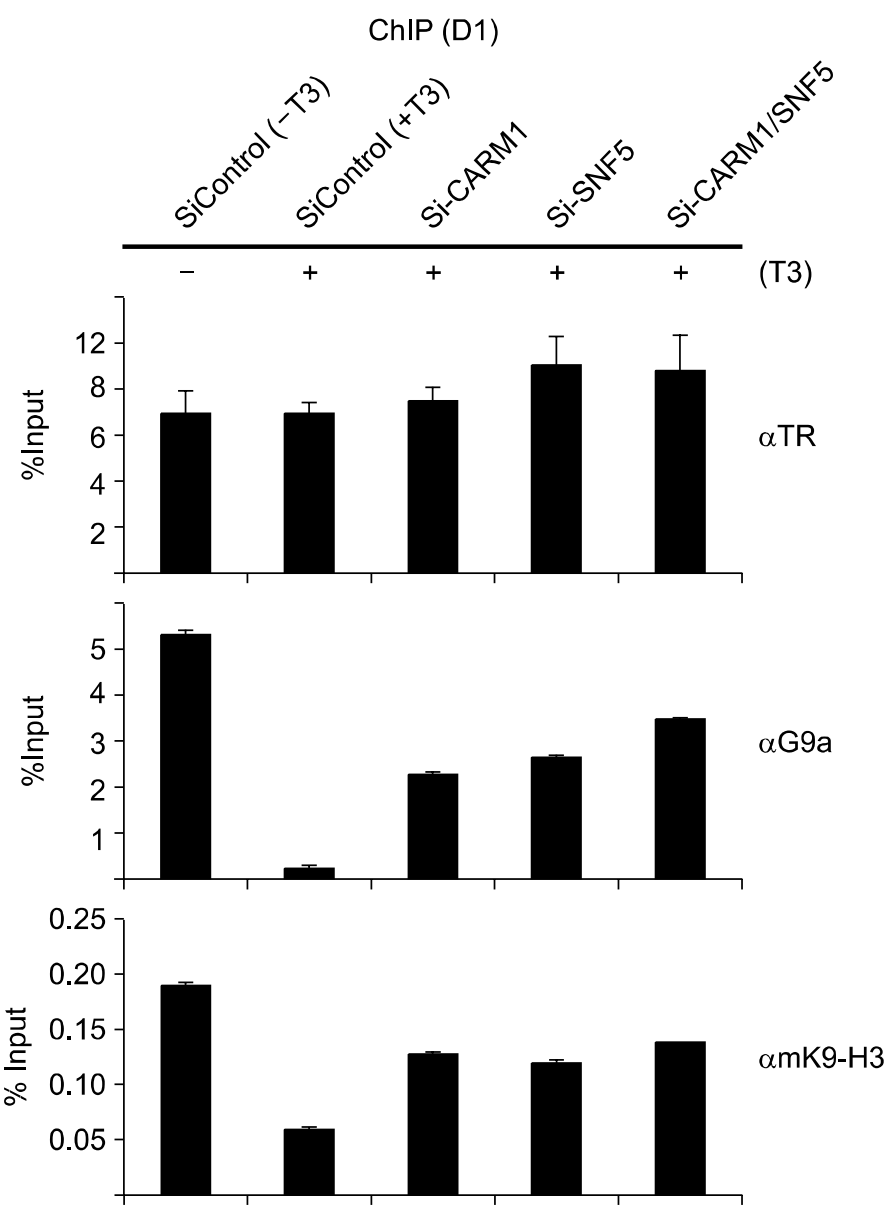

Figure 3. Knocking down of CARM1-SNF5 complex enhances the recruitment of G9a to the D1 promoter even with T3. (A) The binding of cofactors to the D1 promoter was directly tested by ChIP assays using HeLa $\alpha 2$ cells treated with or without T3 for $6 \mathrm{~h}$. (B) HeLa $\alpha 2$ cells were transfected with control (scrambled) siRNA or siRNA against CARM1 and/or SNF5 at the concentration indicated. The cells were then treated with T3 for $6 \mathrm{~h}$, after which the binding of each protein to the D1 promoter were determined by ChIP assays using the antibodies indicated. The results are shown as means \pm SD calculated from three independent experiments.

decrease of methyl K9-H3 (Figure 3B).

\section{The functional importance of the CARM1-SNF5 complex in histone code dynamics}

The CARM1 methyltransferase catalytic function is required for receptor transactivation (Chen et al., 1999) with methylation at R17 of histone H3 serving as a marker of active hormone response elements. Recently, the association of ATPremodeling factors with CARM1 revealed a new arm in the nuclear hormone-signaling cascade (Xu et al., 2004). To study the effect of $m R 17-\mathrm{H} 3$ on the modulation of histone code, we knocked down CARM1 and SNF5 individually or in combination by siRNA and then tested the effect on histone modifications including $\mathrm{mR} 17-\mathrm{H} 3$, acetylation of
$\mathrm{H} 3, \mathrm{mK} 9-\mathrm{H} 3$ and $\mathrm{mH} 2 \mathrm{~A} 2$. As shown in Figure 4, we found that knocking down CARM1 and SNF5 affected the up-regulation of methylation of arginine-17 and acetylation of $\mathrm{H} 3$ as expected. Knocking-down the CARM1-SNF5 complex did not appear to affect the recruitment of TR. The down regulation of $\mathrm{mK} 9-\mathrm{H} 3$ was abolished by treatment with siCARM1 or siSNF5. Interestingly, knockingdown either CARM1 or SNF5 inhibited the downregulation of histone macroH2A, which is correlated with transcriptional activation (Valley et al., 2006). Finally, these data indicate that the functional importance of the CARM1-SNF5 complex in histone code dynamics is correlated with transcriptional activation. 


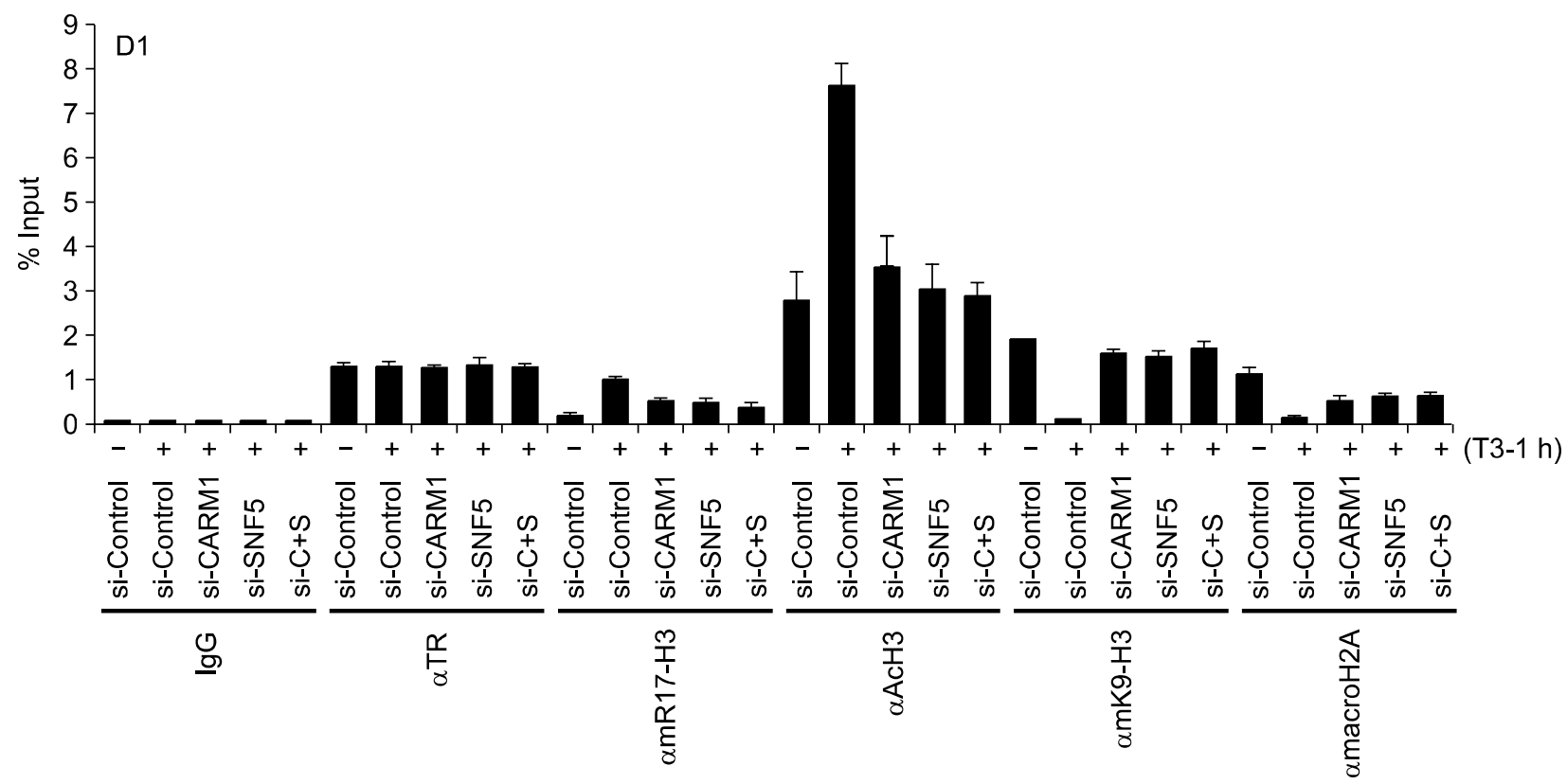

Figure 4. The CARM1-SNF5 complex plays an important role in the functional interplay between histone modifications. The same experiments were carried out as in Figure 2, except that antibodies specific for acetylated TR, methylated arginine 17 of $\mathrm{H} 3$, and methylated $\mathrm{H} 2 \mathrm{~A} 2$ were used.

\section{The CARM1-SNF5 complex is required for the ordered recruitment of coactivators in TR-mediated transcriptional activation}

CARM1 is a coactivator that could have a role in recruiting SWI/SNF. CARM1 interacts with the SRC coactivator family and CBP/p300 (Schurter et al., 2001). In addition, CARM1 has been shown recently to associate with SWI/SNF in a large protein complex termed NUMAC (Xu et al., 2004). Thus, CARM1 could function as a bridging factor between SRC-CBP/p300 and SWI/SNF. To determine if CARM1 and SNF5 affects coactivator recruitment, we used a ChIP assay to determine how knocking down of CARM1 and/or SNF5 affects the recruitment of the following coactivators: members of the SRC family (SRC-1, 2 and 3), CBP/p300, and TRAP/Mediator complex. These coactivators have been well characterized and are known to have critical roles in transcriptional activation by NRs. Furthermore, a recent study showed that all of these coactivators were recruited to the D1 gene promoter by TR (Metivier et al., 2003). We and others have shown by ChIP assay that SRC-1, CBP/p300, the TRAP/Mediator complex and SWI/SNF were recruited to the target promoters by liganded TR (Huang et al., 2003; Yoon et al., 2005). ChIP assay also showed that their recruitment is affected after knocking down CARM1, which had little effect on SRC-2 (Figure $5)$. Whether this result reflects a saturated recruitment of SRC-2 is not known. Together, the
CARM1-SNF5 complex is a key bridging factor required for the ordered recruitment of coactivators in TR-mediated activation.

\section{Discussion}

Accumulated evidence indicates that coactivators CARM1 and the ATP-dependent chromatin remodeling factor SWI/SNF have critical functions in transcriptional activation by nuclear receptors. However, the underlying mechanism for requirement of various chromatin remodeling factors remains obscure. CARM1 cooperates synergistically with the SRC family of coactivators and CBP/p300 to enhance gene activation by NRs as observed in transient transfection assays (Chen et al., 2000). In addition, CARM1 associates with the SWI/SNF complex and this association seems to enhance CARM1's HMT activity. Finally, CARM1 null mice die perinatally and estrogen-responsive gene expression is aberrant in Carm1-/- fibroblasts and embryos, indicating a critical role for CARM1 in mediating transcriptional regulation by estrogen receptors. However, why histone methylation by CARM1 is important for transcription remains to be elucidated.

To investigate the functional importance of coactivators in T3-dependent activation, we first attempted to generate siRNAs against coactivators as indicated (Figure 1). Using these siRNAs, we 


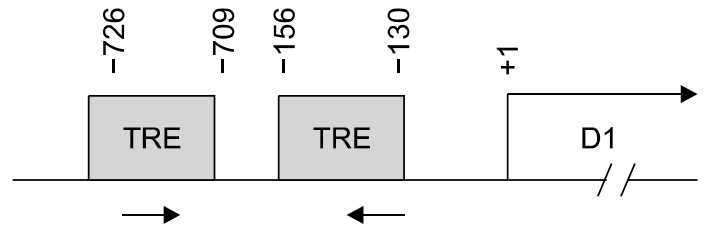

$\mathrm{P} 1$
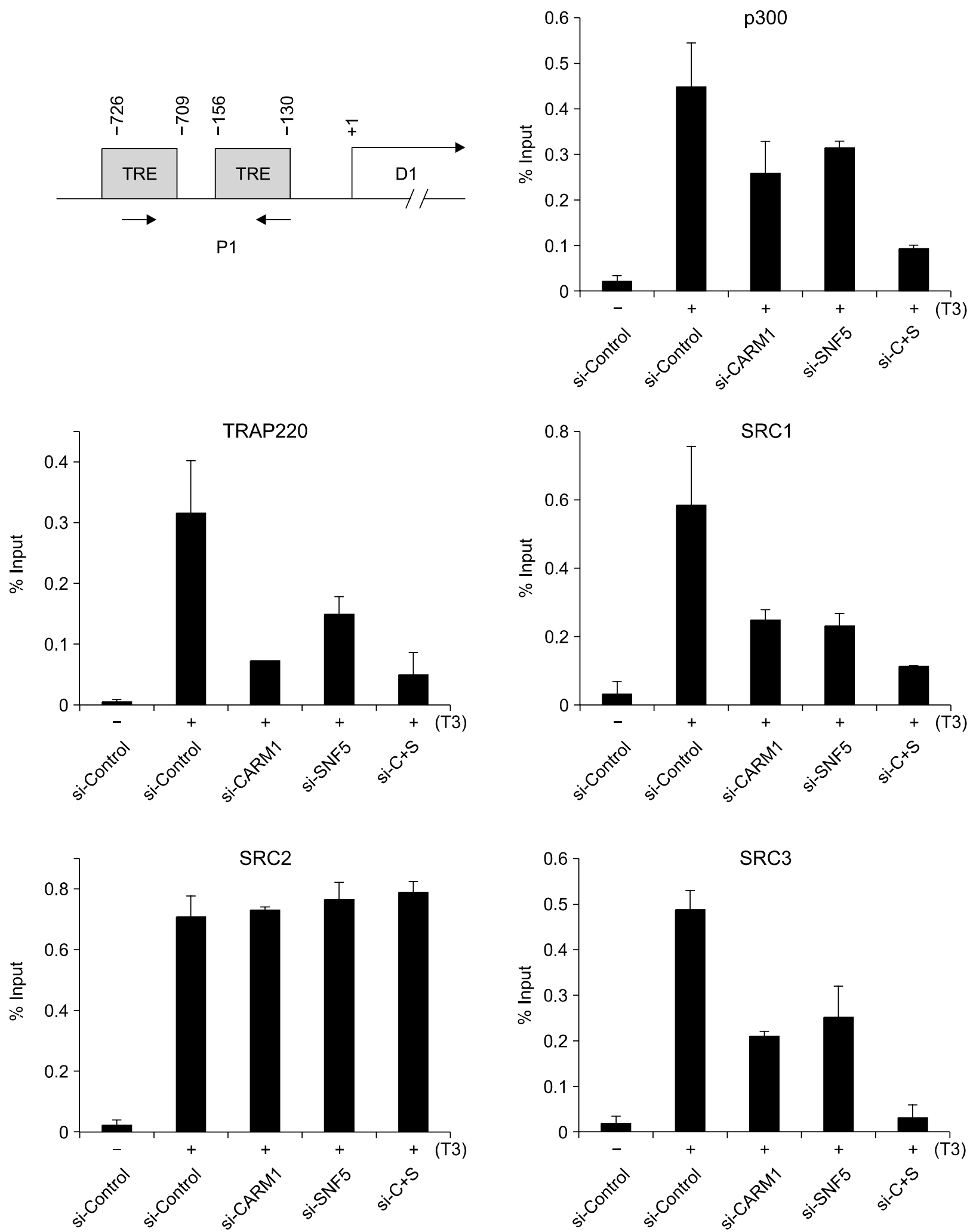

Figure 5. Knocking down of CARM1-SNF5 complex together impaired the chromatin targeting of coactivators by ligand-bound TR. HeLa $\alpha 2$ cells were treated with siRNA against CARM1 and/or SNF5 to knock down their expression individually or in combination. The cells were then treated with T3 (1 nM) for six $h$ and the recruitment of coactivators to the D1 promoter, was then determined by ChIP assays using the antibodies indicated. The results were analyzed by real-time PCR and were shown as the percentage of input. The results are shown as means \pm SD calculated from three independent experiments. 
then analyzed the effect of knocking-down coactivators on D1 expression. Knocking down CARM1 and SNF5 significantly impaired T3-dependent activation by TR. Next, to substantiate whether this result was gene specific or a general mechanism, we tested the effect of knocking down CARM1 and SNF5 on the expression of the other three TR target genes in HeLa $\alpha 2$ cells. As shown in Figure 1C, we found that both CARM1 and SNF5 are generally required for T3-dependent activation. siRNAs against $\mathrm{hBrg} 1$ and $\mathrm{hBrm}$ also reduced T3-dependent activation, but to lesser extent than did siSNF5, presumably because of the functional redundancy between $\mathrm{hBrg} 1$ and $\mathrm{hBrm}$. In addition, CARM1 associates with the SWI/SNF complex and this association seems to enhance CARM1's HMT activity (Xu et al., 2004). Finally, our data indicate that the CARM1-SWI/SNF complex is important for T3-dependent transcriptional activation in mammalian cells.

Histone methylation is known to be stable metabolically (Waterborg, 1993; Kang et al., 2007). The presence of $\mathrm{K} 9$ methylated $\mathrm{H} 3$ ( $\mathrm{mH} 3-\mathrm{K} 9)$ in genes repressed by unliganded TR raises question as to how a liganded receptor overcomes this repressive activity during T3-dependent activation. Surprisingly, as shown in our data (Figure 2), we found by ChIP assay that the level of $\mathrm{mH} 3-\mathrm{K} 9$ in the $\mathrm{D} 1$ promoter decreased rapidly upon $\mathrm{T} 3$ treatment. Here we tested the hypothesis that chromatin remodeling by SWI/SNF serves as a mechanism for the rapid reversal of $\mathrm{H} 3-\mathrm{K} 9$ methylation. Finally, we identified that CARM1SNF5 is required for the rapid reversal of H3-K9 methylation by using siRNAs and ChIP assay. It is unclear why a knocking-down of CARM1 and/or SNF5 increased the level of methyl K9-H3. CARM1 has been shown recently to associate with SWI/SNF in a large protein complex termed NUMAC. In addition, nucleosome unfolding at transcriptionally active promoter by chromatin remodeling complexes have been reported (Boeger et al., 2003; Adkins et al., 2004). Based on these studies, the nucleosome clearance presumably correlates with the change of histone methylation and initiation of transcription. As shown in Figure $3 \mathrm{~B}$, we found that siRNA treatments against CARM1 and/or SNF5 increased the recruitment of HMTase, G9a. The enhanced recruitment of G9a reversed greatly T3-induced decrease of methyl $\mathrm{K} 9-\mathrm{H} 3$. Taken together, we believe the chromatin remodeling by SWI/SNF is likely to be at lease one of the mechanisms for rapid removal of the repressive $\mathrm{mH}$ - $\mathrm{K} 9$ marker during T3-dependent activation, although the involvement of other mechanisms such as demethylation cannot be excluded.

Coactivator that could have a role in recruiting SWI/SNF is CARM1. CARM1 interacts with the SRC family of coactivators and CBP/p300 (Schurter et al., 2001). In addition, CARM1 has been shown recently to associate with SWI/SNF in a large protein complex termed NUMAC. Thus, CARM1 could function as a bridging factor between SRC-CBP/p300 and SWI/SNF. We employed siCARM1 and/or siSNF5 to test whether knocking down CARM1 affects the recruitment of coactivators involved in T3-dependent activation as described. Knocking down the CARM1-SNF5 complex impaired targeting of coactivators to TR target genes, but had little effect on SRC-2. Together, the CARM1-SNF5 complex is important for the recruitment of a coactivator involved in T3-depedent activation.

In summary, we demonstrated that the CARM1 complex is generally required for transcriptional activation by TR. In principle, as transcription is a multi-step process, CARM1 could exert its function at one or more of the essential steps. First, it could be required for the recruitment of other essential coactivators. Second, it could be required for the assembly of the pol II preinitiation complex. Third, it could be required for the transition from transcription initiation to elongation. One limitation in dissecting the role of CARM1 as outlined above is that it would be difficult to pinpoint the exact function of CARM1 even though knocking down CARM1 could have multiple effects on transcription. For instance, as knocking down CARM1 affects coactivator recruitment, it also might affect recruitment of basal transcriptional machinery and/or transcriptional elongation. In this case, the effect on these subsequent steps could be the indirect effect of the coactivator(s) being affected. Despite this limitation, this study should yield significant insight into the mechanism by which the CARM1-SNF5 complex affects transcription.

\section{Acknowledgement}

This study was supported by Korea Research Foundation Grant funded by the Korean Government (MOEHRD) (KRF-2005-042-E00022) and (KRF-2006-331-E00036); (R13-2002-054-01001-0 [2004]) from the Basic Research Program of the Korea Science and Engineering to (H.G. Yoon). This work was supported by the Seoul Science Fellowship to (H.K.Choi).

\section{References}

Adkins MW, Howar SR, Tyler JK. Chromatin disassembly 
mediated by the histone chaperone Asf1 is essential for transcriptional activation of the yeast $\mathrm{PHO} 5$ and $\mathrm{PHO} 8$ genes. Mol Cell 2004;14:657-66

An BS, Ku BJ, Park SY, Shin JK, Lee JH, Kim YK, Shong MH, Ro HK. Hormonal regulation of ICAM-1 gene expression in thyroid cells, FRTL-5. Exp Mol Med 1997;29:45-51

Boeger H, Griesenbeck J, Strattan JS, Kornberg RD. Nucleosome unfold completely at a transcriptionally active promoter. Mol Cell 2003;11:1587-98

Chen D, Ma H, Hong H, Koh SS, Huang SM, Schurter BT, Aswad DW, Stallcup MR. Regulation of transcription by a protein methyltransferase. Science 1999;284:2174-7

Chen DS, Huang SM, Stallcup MR. Synergistic, p160 coactivator-dependent enhancement of estrogen receptor function by CARM1 and p300. J Biol Chem 2000;275: 40810-6

Chiba $\mathrm{H}$, Muramatsu M, Nomoto $\mathrm{A}$, Kato $\mathrm{H}$. Two human homologues of Saccharomyces cerevisiae SWI2/SNF2 and Drosophila brahma are transcriptional coactivators cooperating with the estrogen receptor and the retinoic acid receptor. Nucleic Acids Res 1994;22:1815-20

Dilworth FJ, Fromental-Ramain C, Yamamoto K, Chambon P. ATP-Driven chromatin remodeling activity and histone acetyltransferases act sequentially during transactivation by RAR/RXR in vitro. Mol Cell 2000;6:1049-58

DiRenzo J, Shang Y, Phelan M, Sif S, Myers M, Kingston R, Brown M. BRG-1 is recruited to estrogen-responsive promoters and cooperates with factors involved in histone acetylation. Mol Cell Biol 2000;20:7541-9

Guenther MG, Lane WS, Fischle W, Verdin E, Lazar MA, Shiekhattar R. A core SMRT corepressor complex containing HDAC3 and TBL1, a WD40-repeat protein linked to deafness. Genes Dev 2000;14:1048-57

Heery DM, Kalkhoven E, Hoare S, Parker MG. A signature motif in transcriptional co-activators mediates binding to nuclear receptors. Nature 1997;387:733-6

Huang ZQ, Li J, Sachs LM, Cole PA, Wong J. A role for cofactor-cofactor and cofactor-histone interactions in targeting p300, SWI/SNF and Mediator for transcription. EMBO J 2003;22:2146-55

Kang JK, Park KW, Chung YG, You JS, Kim YK, Lee SH, Hong SP, Choi KM, Heo KN, Seol JG, Lee JH, Jin DI, Park CS, Seo JS, Lee HW, Han JW. Coordinated change of a ratio of methylated $\mathrm{H} 3$-lysine 4 or acetylated $\mathrm{H} 3$ to acetylated $\mathrm{H} 4$ and methylation is associated with tissue-specific gene expression in cloned pig. Exp Mol Med 2007;39:84-96

Kawasaki H, Eckner R, Yao TP, Taira K, Chiu R, Livingston DM, Yokoyama KK. Distinct roles of the co-activators p300 and $\mathrm{CBP}$ in retinoic-acid-induced F9-cell differentiation. Nature 1998;393:284-9

Korzus E, Torchia J, Rose DW, Xu L, Kurokawa R, McInerney EM, Mullen TM, Glass CK, Rosenfeld MG. Transcription factor-specific requirements for coactivators and their acetyltransferase functions. Science 1998;279:703-7

Kraus WL, Manning ET, Kadonaga JT. Biochemical analysis of distinct activation functions in p300 that enhance transcription initiation with chromatin templates. Mol Cell Biol 1999;19:8123-35

Lee YJ, Lim WC, Jin YH, Lee SK. Effects of estrogen receptor and estrogen on the chromatin structure in estrogen receptor stable transfectants. Exp Mol Med 2002;34:95-9

Levine $M$, Tjian $R$. Transcription regulation and animal diversity. Nature 2003;424:147-51

Li J, Lin Q, Yoon HG, Huang ZQ, Strahl BD, Allis CD, Wong $J$. Involvement of histone methylation and phosphorylation in regulation of transcription by thyroid hormone receptor. Mol Cell Biol 2002;22:5688-97

Mclnerney EM, Rose DW, Flynn SE, Westin S, Mullen TM, Krones A, Inostroza J, Torchia J, Nolte RT, Assa-Munt N, Milburn MV, Glass CK, Rosenfeld MG. Determinants of coactivator LXXLL motif specificity in nuclear receptor transcriptional activation. Genes Dev 1998;12:3357-68

McKenna NJ, O'Malley BW. Minireview: nuclear receptor coactivators--an update. Endocrinology 2002;143:2461-5

Metivier R, Penot G, Hubner MR, Reid G, Brand H, Kos M, Gannon F. Estrogen receptor-alpha directs ordered, cyclical, and combinatorial recruitment of cofactors on a natural target promoter. Cell 2003;115:751-63

Naar AM, Lemon BD, Tjian R. Transcriptional coactivator complexes. Annu Rev Biochem 2001;70:475-501

O'Shea PJ, Williams GR. Insight into the physiological actions of thyroid hormone receptors from genetically modified mice. J Endocrinol 2002;175:553-70

Rosenfeld MG, Glass CK. Coregulator codes of transcriptional regulation by nuclear receptors. J Biol Chem 2001;17:17

Schurter BT, Koh SS, Chen D, Bunick GJ, Harp JM, Hanson BL, Henschen-Edman A, Mackay DR, Stallcup MR, Aswad DW. Methylation of histone H3 by coactivator-associated arginine methyltransferase 1 . Biochemistry 2001;40:574756

Strahl BD, Briggs SD, Brame CJ, Caldwell JA, Koh SS, Ma $H$, Cook RG, Shabanowitz J, Hunt DF, Stallcup MR, Allis CD. Methylation of histone $\mathrm{H} 4$ at arginine 3 occurs in vivo and is mediated by the nuclear receptor coactivator PRMT1. Curr Biol 2001;11:996-1000

Valley CM, Pertz LM, Balakumaran BS, Willard HF. Chromosome-wide, allele-specific analysis of the histone code on the human X chromosome. Hum Mol Genet 2006;15: 2335-47

Wang $\mathrm{H}$, Huang ZQ, Xia L, Feng Q, Erdjument-Bromage $\mathrm{H}$, Strahl BD, Briggs SD, Allis CD, Wong J, Tempst $P$, Zhang Y. Methylation of Histone $\mathrm{H} 4$ at Arginine 3 Facilitates Transcriptional Activation by Nuclear Hormone Receptor. Science 2001;31:31

Wang W, Cote J, Xue Y, Zhou S, Khavari PA, Biggar SR, Muchardt C, Kalpana GV, Goff SP, Yaniv M, Workman JL, Crabtree GR. Purification and biochemical heterogeneity of the mammalian SWI-SNF complex. EMBO J 1996;15: 5370-82

Waterborg JH. Dynamic methylation of alfalfa histone H3. J 


\section{Biol Chem 1993;268:4918-21}

Wong J, Shi YB, Wolffe AP. A role for nucleosome assembly in both silencing and activation of the Xenopus TR beta A gene by the thyroid hormone receptor. Genes Dev 1995;9:2696-711

Xu J, Li Q. Review of the in vivo functions of the $\mathrm{p} 160$ steroid receptor coactivator family. Mol Endocrinol 2003;17: 1681-92

Xu W, Cho H, Kadam S, Banayo EM, Anderson S, Yates JR 3rd, Emerson BM, Evans RM. A methylation-mediator complex in hormone signaling. Genes Dev 2004;18:144-56

Yadav N, Lee J, Kim J, Shen J, Hu MC, Aldaz CM, Bedford MT. Specific protein methylation defects and gene expression perturbations in coactivator-associated arginine methyltransferase 1-deficient mice. Proc Natl Acad Sci USA 2003;100:6464-8

Yao TP, Oh SP, Fuchs M, Zhou ND, Ch'ng LE, Newsome D, Bronson RT, Li E, Livingston DM, Eckner R. Gene dosage- dependent embryonic development and proliferation defects in mice lacking the transcriptional integrator p300. Cell 1998;93:361-72

Yi TG, Baek JH, Kim HJ, Choi MH, Seo SB, Ryoo HM, Kim GS, Woo KM. Trichostatin A-mediated upregulation of p21WAF1 contributes to osteoclast apoptosis. Exp Mol Med 2007;39:213-21

Yoon HG, Chan DW, Huang ZQ, Li J, Fondell JD, Qin J, Wong J. Purification and functional characterization of the human N-CoR complex: the roles of HDAC3, TBL1 and TBLR1. EMBO J 2003a;22:1336-46

Yoon HG, Chan DW, Reynolds AB, Qin J, Wong J. N-CoR mediates DNA methylation-dependent repression through a methyl CpG binding protein Kaiso. Mol Cell 2003b;12:723-34 Yoon HG, Choi Y, Cole PA, Wong J. Reading and function of a histone code involved in targeting corepressor complexes for repression. Mol Cell Biol 2005;25:324-35 